\title{
Benign Paraganglioma
}

National Cancer Institute

\section{Source}

National Cancer Institute. Benign Paraganglioma. NCI Thesaurus. Code C48314.

A parag ang lioma that is confined to the site of origin, without metastatic potential. 